\title{
BROOKHISEN
}

NATIONAL LABORATORY

BNL-106312-2014-CP

\section{Virtual Data Center Allocation with Dynamic Clustering in Clouds}

\author{
Li Shi
}

\section{3rd IEEE I nternational Performance Computing and Communications Conference (I PCCC 2014) \\ Austin, TX \\ December 5-7, 2014}

Dec 2014

\author{
Computational Science Center \\ Brookhaven National Laboratory
}

\author{
U.S. Department of Energy \\ Office of Advanced Scientific Computing Reserach
}

Notice: This manuscript has been authored by employees of Brookhaven Science Associates, LLC under Contract No. DE-AC02-98CH10886 with the U.S. Department of Energy. The publisher by accepting the manuscript for publication acknowledges that the United States Government retains a non-exclusive, paid-up, irrevocable, world-wide license to publish or reproduce the published form of this manuscript, or allow others to do so, for United States Government purposes. 
This preprint is intended for publication in a journal or proceedings. Since changes may be made before publication, it may not be cited or reproduced without the author's permission.

\section{DISCLAIMER}

This report was prepared as an account of work sponsored by an agency of the United States Government. Neither the United States Government nor any agency thereof, nor any of their employees, nor any of their contractors, subcontractors, or their employees, makes any warranty, express or implied, or assumes any legal liability or responsibility for the accuracy, completeness, or any third party's use or the results of such use of any information, apparatus, product, or process disclosed, or represents that its use would not infringe privately owned rights. Reference herein to any specific commercial product, process, or service by trade name, trademark, manufacturer, or otherwise, does not necessarily constitute or imply its endorsement, recommendation, or favoring by the United States Government or any agency thereof or its contractors or subcontractors. The views and opinions of authors expressed herein do not necessarily state or reflect those of the United States Government or any agency thereof. 


\section{Virtual Data Center Allocation with Dynamic Clustering in Clouds}

\author{
Li Shi \\ Department of Electrical and \\ Computer Engineering, \\ Stony Brook University, \\ Stony Brook, NY 11794. \\ li.shi@ stonybrook.edu
}

\author{
Dimitrios Katramatos \\ Computational Science Center, \\ Brookhaven National Laboratory, \\ Upton, NY 11973. \\ dkat@bnl.gov
}

\author{
Dantong $\mathrm{Yu}$ \\ Computational Science Center, \\ Brookhaven National Laboratory, \\ Upton, NY 11973. \\ dtyu@bnl.gov
}

\begin{abstract}
Clouds are being widely used for leasing resources to users in the form of on-demand virtual data centers, which comprise sets of virtual machines interconnected by sets of virtual links. Given a user request for a virtual data center with specific resource requirements, a critical problem is to select a set of servers and links in the physical data center of a cloud to satisfy the request in a manner that minimizes the amount of reserved resources. In this paper, we study the main aspects of this Virtual Data Center Allocation (VDCA) problem, and decompose it into three subproblems: virtual data center clustering, virtual machine allocation, and virtual link allocation. We prove the NP-hardness of VDCA and propose an algorithm that solves the problem by dynamically clustering the requested virtual data center and jointly optimizing virtual machine and virtual link allocation. We further compare the performance and scalability of the proposed algorithm with two existing algorithms, called LoCo and SecondNet, through simulations. We demonstrate that our algorithm generates $30 \%-200 \%$ more revenue than LoCo and $55 \%-300 \%$ than SecondNet, while being up to 12 times faster.
\end{abstract}

\section{INTRODUCTION}

Clouds are being widely used to provide the service of leasing resources to users. In clouds, such as Amazon EC2 [1] and Microsoft Azure [2], Service Providers (SPs) own a number of data centers and through cloud technologies lease both computing and network resources to users in the form of ondemand Virtual Data Centers (VDCs) [3]. A VDC is composed by a set of virtual machines (VMs) interconnected by a set of virtual links. Through modern virtualization techniques, a SP is able to simultaneously provision VDCs for multiple users while keeping the provisioned VDCs isolated - and interference-free - from each other. Many Quality of Service (QoS) mechanisms [3]-[6] have been proposed to provide bandwidth guarantees to provisioned VDCs. Thanks to such QoS mechanisms, users are able to execute applications in their own VDC with guaranteed and predictable performance. Given a user request for a VDC with certain resource requirements for both VMs and virtual links, an important problem is to find a set of servers and links in the data center to satisfy the request. We call this problem the Virtual Data Center Allocation (VDCA) problem. At first glance, the VDCA problem seems similar to the Virtual Network Embedding (VNE) problem, which refers to the ways of mapping a virtual network that connects a set of VMs onto a substrate network that connects a set of geographically distributed servers. However, the VDCA problem is significantly different than the VNE problem.

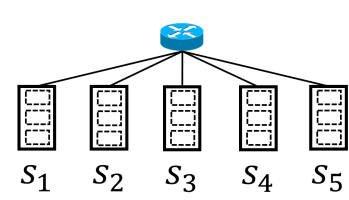

(a)

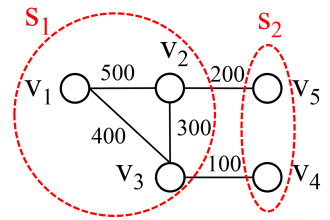

(b)
Fig. 1. An example of two different clustering schedules.

First, in contrast to allocating (mapping) virtual nodes on physical nodes in a one-on-one fashion in the VNE problem [7]-[11], SPs of clouds usually try to collocate multiple VMs requested by the same user on the same host(s). By doing so, SPs do not need to reserve bandwidth on physical network links for the virtual links that connect the collocated VMs. Therefore, such VM collocation essentially reduces the amount of available resources reserved for each user request ${ }^{1}$. As a result, a cloud can potentially service more users and thereby generate more revenue. For example, Fig. 1(a) shows a small data center with five servers and Fig. 1(b) shows a VDC request. Assuming that each server can host three VMs, if we allocate VMs with one-on-one mapping, we then need to find paths and reserve bandwidth for all virtual links with a total bandwidth requirement of 2600 Mbps. However, if we allocate VMs $v_{1}, v_{2}$ and $v_{3}$ together on server $s_{1}$ and allocate VMs $v_{4}$ and $v_{5}$ on server $s_{2}$. we then only need to reserve bandwidth for virtual links $\left(v_{2}, v_{5}\right)$ and $\left(v_{3}, v_{4}\right)$ with a total bandwidth requirement of $300 \mathrm{Mbps}$. To fully exploit VM collocation, one faces the novel and critical problem of how to collocate VMs in a manner that minimizes the total requirement of bandwidth to be reserved on the physical links, while considering the availability of resources of both servers and links. If we consider the VMs allocated on the same host as a cluster of the VDC, this problem then becomes a problem of clustering the VDC. As a result, we can decompose the VDCA problem into three subproblems: VDC clustering, VM allocation, and virtual link allocation.

Additionally, in the VNE problem, the substrate network usually comprises tens to hundreds of servers and a virtual network request usually includes tens of VMs [7]-[11]. In contrast, in the VDCA problem, the data centers can comprise

\footnotetext{
${ }^{1} \mathrm{We}$ assume that the internal bandwidth of a server is always enough to satisfy the bandwidth requirement of all virtual links that interconnect VMs on that server [4], [12].
} 
thousands of servers and a VDC request can include hundreds of VMs. For example, traces of workloads running on an 12000-machine Google compute cell [13] has shown that the number of machines required by user jobs can be as large as 2000, while the average number of jobs submitted every day is larger than 4000. These aspects of the VDCA problem impose significant scalability requirements on the scheduling algorithm.

In this paper, we study the VDCA problem. We formally formulate the problem and prove it is NP-hard, then propose an algorithm, called the Virtual Data Center Allocation (ViDCAlloc) algorithm, to solve it. The ViDCAlloc algorithm dynamically clusters a VDC and jointly optimizes VM and virtual link allocation. ViDCAlloc incorporates several problemspecific techniques, such as Virtual Cluster Expansion and New Virtual Cluster Establishment. We study the performance and scalability of the ViDCAlloc algorithm through a set of simulations.

In section II we describe related work, while in section III we formally define the problem. In section IV we present the proposed algorithm and analyze its runtime complexity, then in section $\mathrm{V}$ we study the performance of our algorithm through simulations. Finally, in section VI we present our conclusions.

\section{RELATED WORK}

A significant amount of research has focused on cloud computing and virtual network embedding. In this section, we discuss some of the research works that we consider most relevant to our problem.

The virtual network embedding (VNE) problem has been well studied [7]-[11]. Fischer et al. [7] give a comprehensive survey of the VNE problem. Chowdhury et al. [8] formulate VNE as a Mixed Integer Programming (MIP) problem, solve its linear programming relaxation and then use a heuristic to determine a final solution. Yu et al. [9] proposes a VNE approach that maximizes long-term revenue. Lischka et al. [10] reduce the VNE problem to the well known Subgraph Isomorphism Detection (SID) problem, and propose a modification of the existing heuristic to solve it. Gong et al. [11] propose a novel metric, called global resource capacity (GRC), to quantify the embedding potential of each substrate node. Gong et al. further develop an algorithm, called GRC-VNE, to solve the VNE problem by utilizing the GRC metric. However, all of these proposed approaches allocate virtual nodes on physical nodes in a one-on-one mapping manner. Without benefiting from collocating VMs, the efficiency of these approaches in solving the VDCA problem may be diminished. In addition, most of these approaches have high complexity, which limits their scalability in solving large scale problems.

In the context of cloud computing, Fuerst et al. [12] proposes an approach that solves the VDCA problem in two steps. In the first step, a pre-clustering approach is used to cluster the requested VDC and generate a new clustered VDC request. Next, a collocation algorithm, called LoCo, is developed to service the clustered VDC request. The preclustering approach requires an accurate estimation of the available data center resources and its complexity is high. In contrast to [12], our proposed algorithm performs clustering and allocation jointly. We compare our algorithm with [12]

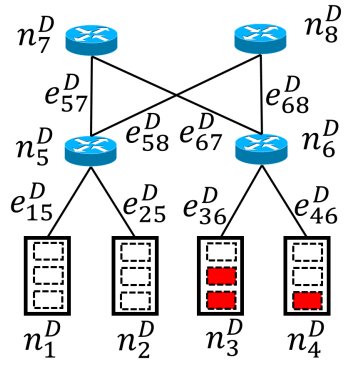

(a) Topology of graph $G^{D}$

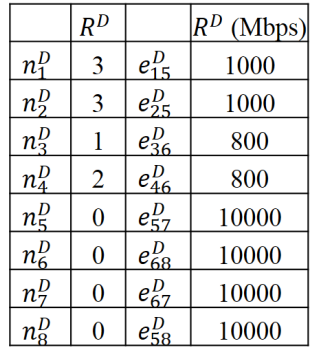

(b) $R^{D}$ of $G^{D}$
Fig. 2. An example of input $G^{D}$.

through simulations. Papagianni et al. [14] consideres the problem of allocating resources in a networked cloud and formulates it as a MIP problem, then proposes a heuristic approach to solve it. However, the formulated MIP problem also enforces one-on-one mapping between VMs and servers. Guo et al. [3] develops an algorithm to map the VDC request onto the physical data center. However, the proposed algorithm again allocates VMs in a one-one-one mapping manner. We also compare our algorithm with the algorithm proposed in [3]

\section{PROBLEM DESCRIPTION}

We consider the physical data center of a cloud that incorporates a set of servers and switches. Assume that the SP of this cloud receives a VDC request which includes a set of VMs and virtual links. Given the topology and resource availability of the data center, our goal is to find a feasible allocation schedule for the request while minimizing a desired objective function. We can formally describe the input, output, and objective function of the algorithm as follows:

Input: The input has three components. The first component is the topology and resource availability of the physical data center. We model it as a graph $G^{D}=\left(N^{D}, E^{D}, R^{D}\right)$, where $N^{D}$ is the set of all nodes, in which each server or switch corresponds to one node; $E^{D}$ is the set of all physical links, in which each $e_{i j}^{D}$ represents the physical link that connects nodes $n_{i}^{D}$ and $n_{j}^{D}$; and $R^{D}$ is the set containing the resource availability of each server and each link. In this paper, we consider as schedulable resources the computing capacity of a server and the bandwidth of a link, following the model used in [8], [9], [11], [12]. Because in VM hypervisors, like Xen [15] and KVM [16], the computing capacity of a VM is usually defined by the number of CPU cores reserved for that VM, we model the available computing capacity of a server as the number of its available CPU cores. Specifically, $r\left(n_{i}^{D}\right) \in R^{D}$ represents the available CPU cores of node $n_{i}^{D}$, and $r\left(e_{i j}^{D}\right) \in R^{D}$ represents the available bandwidth of link $e_{i j}^{D}$. If node $n_{i}^{D}$ is a switch, we then set $r\left(n_{i}^{D}\right)$ to zero. Fig. 2 shows an example of graph $G^{D}$, which includes eight nodes (four servers and four switches) and eight links. Observe that some CPU cores of nodes $n_{3}^{D}$ and $n_{4}^{D}$ have already been reserved for previously allocated VMs (red rectangles).

The second component is the submitted VDC request. We model such a request as a graph $G^{V}=\left(N^{V}, E^{V}, R^{V}\right)$, where $N^{V}$ is the set of VMs; $E^{V}$ is the set of virtual edges ${ }^{2}$ that

\footnotetext{
${ }^{2}$ In this paper, we interchangeably use the terms virtual link and virtual edge.
} 


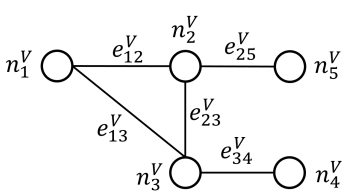

(a) Topology of graph $G^{V}$.

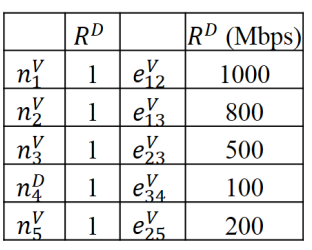

(b) $R^{V}$ of $G^{V}$.
Fig. 3. An example of input $G^{V}$.

interconnect the VMs, and each $e_{i j}^{V}$ represents the virtual edge (VE) that connects $n_{i}^{V}$ and $n_{j}^{V}$; and $R^{V}$ is the set of resource requirements of each VM and VE. $r\left(n_{i}^{V}\right) \in R^{V}$ represents the number of CPU cores required by the VM $n_{i}^{V}$, and $r\left(e_{i j}^{V}\right) \in$ $R^{V}$ represents the amount of bandwidth required by the $\mathrm{VE}$ $e_{i j}^{V}$. Fig. 3(a) shows an example of $G^{V}$ containing five VMs and four VEs.

Output: The output of the algorithm contains two components. The first component is the VM allocation schedule, which is denoted by:

$$
M^{N}: N^{V} \rightarrow N^{D}
$$

in which $M^{N}\left(n_{i}^{V}\right)$ is the node that accommodates VM $n_{i}^{V}$. The mapping $M^{N}$ is subject to the constraint that the total number of CPU cores required by the VMs allocated on a physical node cannot exceed the number of available CPU cores of that node. This constraint can be expressed as:

$$
\sum_{\substack{\forall n_{i}^{V}, \text { where } \\ M^{N}\left(n_{i}^{V}\right)=n_{j}^{D}}} r\left(n_{i}^{V}\right) \leq r\left(n_{j}^{D}\right), \quad \forall n_{j}^{D} \in N^{D}
$$

The second component is the virtual edge allocation schedule, which is denoted by:

$$
\begin{gathered}
M^{E}: E_{C}^{V} \rightarrow P^{D} \\
\text { where } E_{C}^{V}:\left\{e_{i j}^{V} \mid M^{N}\left(n_{i}^{V}\right) \neq M^{N}\left(n_{j}^{V}\right)\right\}
\end{gathered}
$$

in which $M^{E}\left(e_{i j}^{V}\right)$, also denoted as $p_{V_{i j}}^{D}$, is the physical path allocated for $e_{i j}^{V}$. Each $p_{V_{i j}}^{D}$ contains a set of physical links that form a path from $M^{N}\left(n_{i}^{V}\right)$ to $M^{N}\left(n_{j}^{V}\right)$. The mapping $M^{E}$ is subject to the constraint that the total bandwidth reserved on a physical link cannot exceed the available bandwidth of that link. We can express this constraint as:

$$
r\left(e_{p q}^{D}\right) \geq \sum_{\substack{e_{i j}^{V}, \text { where } \\ e_{p q}^{D} \in M^{E}\left(e_{i j}^{V}\right)}} r\left(e_{i j}^{V}\right), \quad \forall e_{p q}^{D} \in E^{D}
$$

Objective: Our goal is to generate a feasible allocation schedule for the input request while minimizing the total amount of bandwidth reserved in the physical network. We can express the objective function as:

$$
\text { Minimize } \sum_{e_{i j}^{V} \in E_{C}^{V}} \sum_{e_{p q}^{D} \in M^{E}\left(e_{i j}^{V}\right)} r\left(e_{i j}^{V}\right)
$$

We claim that an SP can accommodate more user requests and generate more revenue by minimizing the above objective function for each request. Additionally, we seek to develop an algorithm that has low runtime complexity, and therefore good runtime performance when addressing large scale problems.

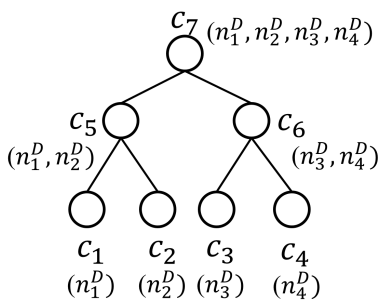

(a)

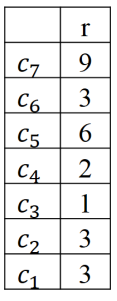

(b)
Fig. 4. (a) The DCC-Tree of the data center shown in Fig. 2(a), (b) the resource availability of each node in the DCC-Tree shown in Fig. 4(a).

Problem Complexity: We first prove that a special instance of our problem, called simple-VDCA, is an NP-hard problem. In simple-VDCA, the user request only contains a set of VMs and their corresponding resource requirements. Given such a user request, our goal is to find a feasible VM allocation schedule $M^{N}$, subject to the constraint of Eq. 2. We now consider the bin packing problem that is known to be NP-hard [17]. In the bin packing problem, given $\mathrm{n}$ bins with their corresponding capacity and $\mathrm{m}$ items with their corresponding size, the goal is to determine a feasible assignment of items to bins such that the sum of the size of items in each bin does not exceed the bin's capacity. We can easily convert the bin packing problem to the simple-VDCA problem by considering the bins as the physical nodes and the items as the VMs. As a result, the simple-VDCA problem is NP-hard. Since the VDCA problem is a generalized version of the simple-VDCA problem, the VDCA problem is also NP-hard.

\section{The Virtual Data Center Allocation (ViDCAlloc) Algorithm}

We develop the ViDCAlloc algorithm to solve the VDCA problem. In this section, we first introduce a pre-processing technique, called Data Center Clustering Tree, that facilitates the VDC allocation. We then describe the ViDCAlloc algorithm and analyze its runtime complexity.

\section{A. Pre-processing: Data Center Clustering Tree}

A Data Center Clustering Tree (DCC-Tree) is a hierarchical clustering tree of the data center. Each node $c_{i}$ in the DCC-Tree represents a cluster that contains a set of servers. The cluster represented by a node is composed by the clusters represented by the child nodes of that node. We can build the DCC-Tree in two steps, as follows: First, for each physical server, we create a cluster node that only contains that server. These created nodes are the nodes at the lowest level (level 0) of the DCC-Tree. Second, we recursively build the upper levels of the DCC-Tree. Specifically, for any level $\mathrm{k}$ that is already built, we group the cluster nodes of that level in a manner that any two servers belonging to two different cluster nodes have the same distance (number of hops). For each group, we then create a new node and set all nodes in that group as child nodes of this new node. Essentially, the new node represents a larger cluster that contains all servers in each of its children nodes. Together, all created parent nodes form the $\mathrm{k}+1$ level of the DCC-Tree. If we create only one node at some level, that node is then the root node of the DCC-Tree and the procedure of building the DCC-Tree finishes. We further define the resource availability of a cluster node as the sum of the resource availabilities of 


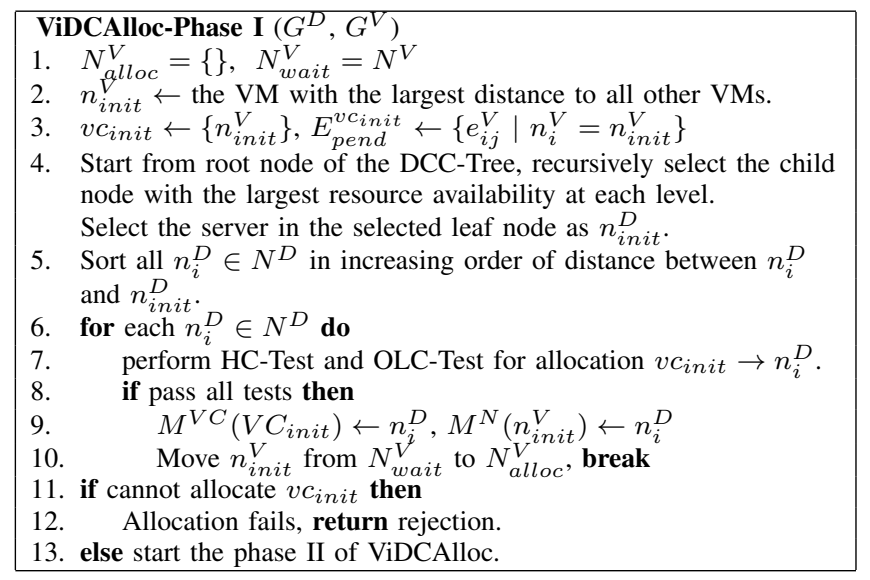

Fig. 5. Pseudocode of phase I of the ViDCAlloc algorithm.

all servers in that cluster node. We express this as:

$$
r\left(c_{i}\right)=\sum_{n_{j}^{D} \in c_{i}} r\left(n_{j}^{D}\right)
$$

where $r\left(c_{i}\right)$ is the resource availability of cluster node $c_{i}$. As an example, Fig. 4(a) shows the DCC-Tree of the data center shown in Fig. 2 and Fig. 4(b) shows the resource availability of each node in this DCC-Tree.

\section{B. Algorithm Overview}

The ViDCAlloc algorithm allocates the requested VDC by grouping the VMs of the VDC into multiple clusters and then allocating each cluster. Because each node of such a cluster represents a VM, we call these clusters Virtual Clusters (VCs). A $\mathrm{VC} v c_{i}$ is a set of VMs and is allocated on some server, denoted by $M^{V C}\left(v c_{i}\right)$. ViDCAlloc also allocates paths for the cross-VC VEs that connect VMs belonging to two different VCs. For example, Fig. 1(a) shows two VCs and two cross-VC VEs: $\left(v_{2}, v_{5}\right)$ and $\left(v_{3}, v_{4}\right)$. Given the objective function shown in Eq. 5, the ViDCAlloc algorithm pursues two goals: (a) to minimize the overall bandwidth requirements of all cross-VC VEs, (b) to minimize the length (number of hops) of paths allocated for cross-VC VEs.

The ViDCAlloc algorithm consists of two phases. In the first phase, the algorithm creates the initial VC and utilizes the DCC-Tree to allocate the initial VC on some server. In the second phase, the algorithm runs in iterations. In each iteration, ViDCAlloc either expands an existing VC by adding an unallocated VM to it, or creates a new VC for an unallocated VM. The pseudocode for these two phases is shown in Fig. 5 and Fig. 7 respectively.

\section{Algorithm Details}

1) Phase I: Set up the initial VC: In this phase, ViDCAlloc creates the first $\mathrm{VC} v c_{\text {init }}$ which only contains one $\mathrm{VM}$ and utilizes the DCC-Tree to allocate $v c_{i n i t}$ on some server. Before introducing more details for this phase, we need to define several items. First, we define set $N_{\text {alloc }}^{V}$ as the set of VMs that have been already allocated and set $N_{\text {wait }}^{V}$ as the set of VMs that have not been allocated yet. We then define set $E_{\text {pend }}^{v c_{i}}$ of $v c_{i}$ as the set of VEs that connect a VM node in $v c_{i}$ with an unallocated VM node in $N_{\text {wait }}^{V}$. Finally, we define as the length

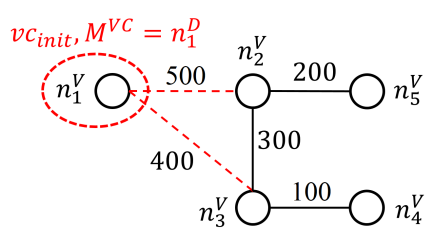

Fig. 6. An example of the inital $\mathrm{VC} v c_{i n i t}$.

of a $\operatorname{VE~} e_{i j}^{V}$ the bandwidth requirement of $e_{i j}^{V}$, i.e., $r\left(e_{i j}^{V}\right)$, and define the distance between two VMs as the total length of the shortest path between them. For example, given input $G^{V}$ shown in Fig. 3, the distance between $n_{1}^{V}$ and $n_{4}^{V}$ is 500 .

The ViDCAlloc algorithm begins by putting all nodes in set $N^{V}$ into $N_{\text {wait }}^{V}$ and leaving set $N_{\text {alloc }}^{V}$ empty. It then selects the VM that has the largest overall distance to all other VMs, denoted by $n_{\text {init }}^{V}$. Next, ViDCAlloc creates $v c_{\text {init }}$ that only contains VM $n_{\text {init }}$ and constructs set $E_{\text {pend }}^{v c_{\text {init }}}$. Subsequently, the algorithm allocates $v c_{\text {init }}$ on a physical server using the following procedure: First, it selects a server $n_{\text {init }}^{D}$ by walking down the DCC-Tree; specifically, it starts from the root node of the DCC-Tree and recursively selects the child node with the largest resource availability at each level; the algorithm stops when it reaches a leaf node and selects the physical server in that node as $n_{\text {init }}$. Next, ViDCAlloc sorts all physical nodes in increasing order of distance (number of hops) between the nodes and $n_{\text {init }}^{D}$. If some nodes have the same distance to $n_{i n i t}^{D}$, the algorithm sorts them in decreasing order of their resource availability. Then, for each node $n_{i}^{D}$, ViDCAlloc examines the feasibility of allocating $v c_{\text {init }}$ on that node by performing two tests, called Host Capacity Test and Outgoing Link Capacity Test.

Host Capacity Test (HC-Test): In this test, for an allocation $v c_{t} \rightarrow n_{i}^{D}$, the ViDCAlloc algorithm checks whether the number of available CPU cores on $n_{i}^{D}$ is larger than or equal to the overall number of CPU cores required by all VMs in $v c_{t}$. Note that the initial VC $v c_{i n i t}$ is $v c_{t}$ in the current step.

Outgoing Link Capacity Test (OLC-Test): In this test, for an allocation $v c_{t} \rightarrow n_{i}^{D}$, the ViDCAlloc algorithm checks whether the outgoing links of $n_{i}^{D}$ have enough available bandwidth to accommodate the VEs in $E_{\text {pend }}^{v c_{t}}$. Assume that node $n_{i}^{D}$ has $M$ outgoing links with available bandwidth $b w_{i}$ and $E_{\text {pend }}^{v c_{t}}$ contains $N$ VEs with bandwidth requirement $r_{j}$. The test then becomes a bin packing problem by considering each outgoing link as a bin and each VE in $E_{\text {pend }}^{v c_{t}}$ as an item. The ViDCAlloc algorithm uses the best fit decreasing strategy [18] to solve this problem. If the ViDCAlloc algorithm finds a feasible assignment, the allocation $\left(v c_{t}, n_{i}^{D}\right)$ passes this test. Similarly, in the current step, the inital $\mathrm{VC} v c_{\text {init }}$ is $v c_{t}$.

If an allocation $v c_{i n i t} \rightarrow n_{i}^{D}$ passes both tests, ViDCAlloc allocates $v c_{\text {init }}$ on $n_{i}^{D}$, i.e., set $M^{V C}\left(v c_{i n i t}\right)$ and $M^{N}\left(n_{\text {init }}^{V}\right)$ as $n_{i}^{D}$, and moves $n_{i n i t}^{V}$ from $N_{\text {wait }}^{V}$ to $N_{\text {alloc }}^{V}$. ViDCAlloc then finishes phase I and starts its next phase. If any of these two test fails, ViDCAlloc tries to allocate $v c_{\text {init }}$ on the next node in $N^{D}$. If ViDCAlloc cannot allocate $v c_{\text {init }}$ on any node in $N^{D}$, it stops and rejects the input request.

As an example, given the input shown in Fig. 2 and Fig. 3, the ViDCAlloc algorithm begins by selecting $n_{1}^{V}$ as $n_{\text {init }}^{V}$. It then sets up $v c_{\text {init }}$ as shown in Fig. 6 and constructs $E_{\text {pend }}^{v c_{i n i t}}$ 


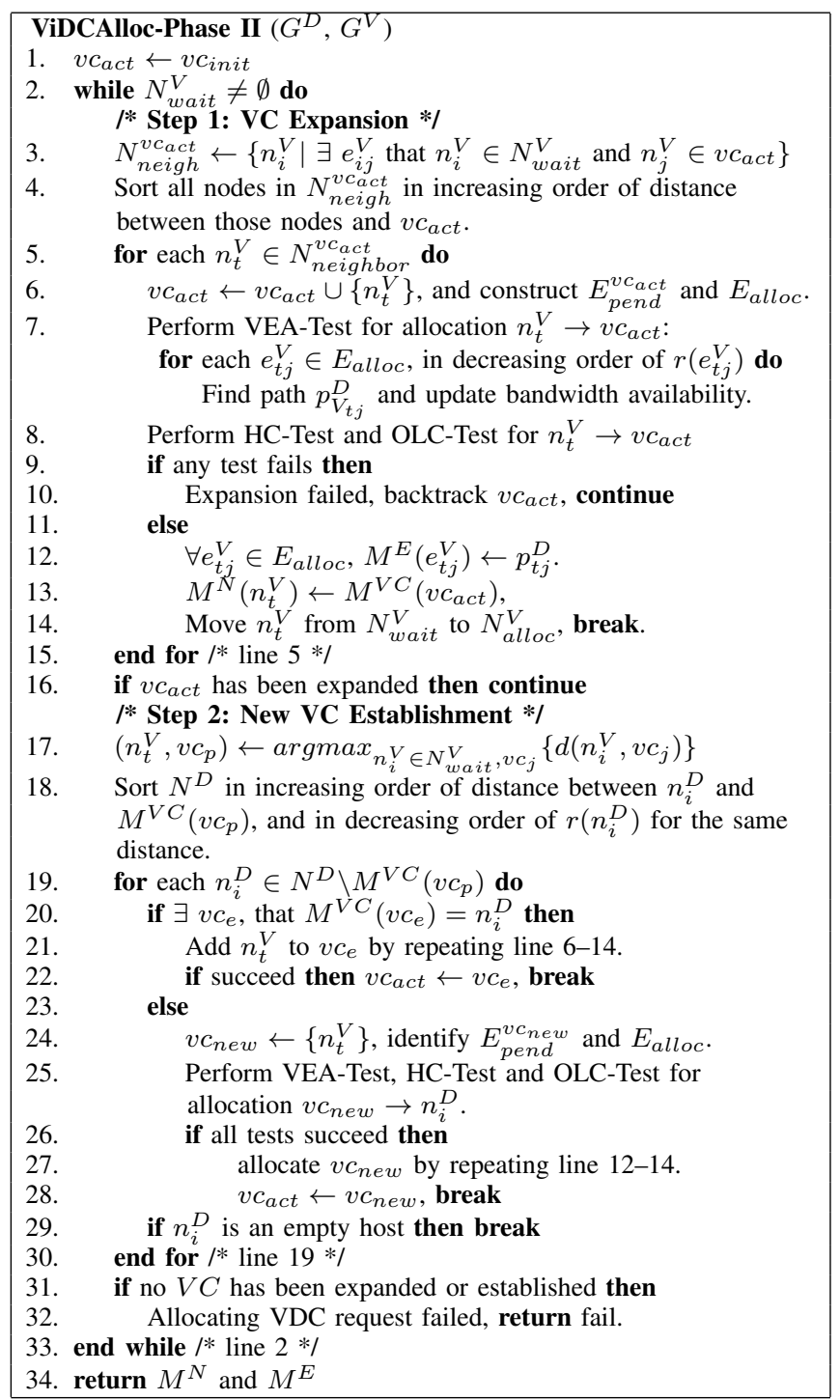

Fig. 7. Pseudocode of phase II of the ViDCAlloc algorithm.

as $\left\{e_{12}^{V}, e_{13}^{V}\right\}$ (dashed red lines). Next, it chooses $n_{1}^{D}$ as $n_{\text {init }}^{D}$ by walking down the DCC-Tree shown in Fig. 4(a) and tries to allocate $v c_{\text {init }}$ on each server node $n_{i}^{D}$ in the sequence $\left(n_{1}^{D}, n_{2}^{D}, n_{4}^{D}, n_{3}^{D}\right)$. The algorithm then performs HC-Test and OLC-Test for each possible allocation. Finally, because the first allocation $v c_{\text {init }} \rightarrow n_{1}^{D}$ passes both tests, the ViDCAlloc algorithm allocates $v c_{\text {init }}$ on node $n_{1}^{D}$ and starts phase II.

2) Phase II: Allocate VDC in iterations: In this phase, the ViDCAlloc algorithm runs in iterations. In each iteration, it allocates one VM in two steps: the VC Expansion (VCE) step and the New VC Establishment (NVCE) step.

Step 1: VC Expansion (VCE) step. In this step, our goal is to expand an existing $\mathrm{VC}$ by adding an unallocated $\mathrm{VM}$ to it. Before introducing the VCE step, we first define the neighbor nodes of a VC $v c_{i}$ as the VM nodes that are in set $N_{\text {wait }}^{V}$ and connected to $v c_{i}$ by one or more VEs. We denote the set of the neighbor nodes of $v c_{i}$ by $N_{n e i g h}^{v c_{i}}$. We further define the distance between $v c_{i}$ and its neighbor node $n_{j}^{V}$ as the sum of the bandwidth requirement of each VE connecting $n_{j}^{V}$ and $v c_{i}$, denoting this by $d\left(n_{j}^{V}, v c_{i}\right)$. For example, given the $\mathrm{VC} v c_{i n i t}$ shown in Fig. 6, set $N_{n e i g h}^{v c_{\text {init }}}$ is $\left\{n_{2}^{V}, n_{3}^{V}\right\}$, while $d\left(n_{2}^{V}, v c_{\text {init }}\right)$

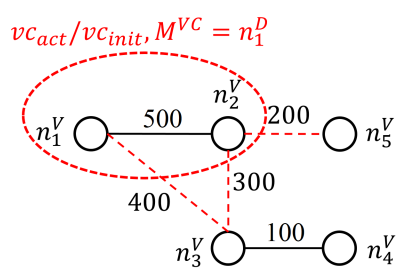

Fig. 8. After the $1^{\text {st }}$ iteration of Phase II of the ViDCAlloc algorithm.

and $d\left(n_{3}^{V}, v c_{\text {init }}\right)$ are 500 and 400 respectively.

In the VCE step, the ViDCAlloc algorithm begins by selecting an existing $\mathrm{VC}$ as the active $\mathrm{VC}$, denoted by $v c_{a c t}$. Then, it constructs set $N_{n e i g h}^{v c_{a c t}}$ and tries to expand $v c_{a c t}$ by allocating one of the nodes in $N_{n e i g h}^{v c_{a c t}}$ to $v c_{a c t}$. Specifically, ViDCAlloc sorts the nodes in $N_{n e i g h}^{v c_{a} \text { igt }}$ in decreasing order of distance between those nodes and VC $v c_{a c t}$, then sequentially checks the feasibility of each allocation $n_{t}^{V} \rightarrow v c_{a c t}$ using the following procedure: First, the algorithm adds node $n_{t}^{V}$ to $v c_{a c t}$ and constructs a new $E_{\text {pend }}^{v c_{a c t}}$. Next, it identifies all VEs that connect $n_{t}^{V}$ to another VM node that is already allocated in an existing $\mathrm{VC}$, and adds them into a set denoted by $E_{\text {alloc. }}$ If $n_{t}^{V}$ is actually allocated to $v c_{a c t}$, these VEs are the cross-VC VEs that require reservation of bandwidth on a physical path which comprises a set of physical network links interconnecting servers. After constructing $E_{\text {alloc }}$, the ViDCAlloc algorithm performs a Virtual Edge Allocation Test, an $H C$-Test and an OLC-Test for allocation $n_{t}^{V} \rightarrow v c_{a c t}$.

Virtual Edge Allocation Test (VEA-Test): In this test, ViDCAlloc sorts the VEs in $E_{\text {alloc }}$ in decreasing order of bandwidth requirements. Then, for each $\mathrm{VE} e_{t j}^{V} \in E_{a l l o c}$, ViDCAlloc finds a physical path between server $M^{V C}\left(v c_{a c t}\right)$ and server $M^{N}\left(n_{j}^{V}\right)$. We define the available bandwidth of a physical path $p$ as the smallest available bandwidth of the physical links in the path and denote it by $B W_{p}$. Clearly, a valid path for a VE $n_{t j}^{V}$ must satisfy the condition $B W_{p} \geq$ $r\left(n_{t j}^{V}\right)$. For each VE $e_{t j}^{V}$, ViDCAlloc then finds a valid shortest path $p_{V_{t j}}^{D}$ with the lowest $B W_{p}$ between $M^{V C}\left(v c_{a c t}\right)$ and $M^{N}\left(n_{j}^{V}\right)$, by using a slightly modified version of Dijkstra's Algorithm [19]. Specifically, the modified Dijkstra's algorithm updates the shortest path from source node $s$ to a node $v$ to the shortest path from $s$ to a node $u$ plus the link connecting $u$ and $v$, if the following constraints are satisfied: (a) The available bandwidth of the link connecting $u$ and $v$ is larger than or equal to $r\left(n_{t j}^{V}\right)$. (b) The length (number of hops) of the new path is shorter than that of the original path; if the length of the two paths is the same, the available bandwidth of the new path is smaller than that of the original path. If ViDCAlloc successfully finds a physical path for each VE in $E_{\text {alloc }}$, the test succeeds; the test otherwise fails.

If an allocation $n_{t}^{V} \rightarrow v c_{a c t}$ passes all tests, the ViDCAlloc algorithm actually allocates $n_{t}^{V}$ to $v c_{a c t}$, i.e., allocates $n_{t}^{V}$ on server $M^{V C}\left(v c_{a c t}\right)$. Additionally, for each $e_{t j}^{V} \in E_{a l l o c}$, the algorithm reserves the required amount of bandwidth (i.e., $r\left(e_{t j}^{V}\right)$ ) on each physical link in the physical path $p_{V_{t j}}^{D}$ found in the VEA-Test step. If any of these three tests fails, ViDCAlloc tries to allocate the next node in $N_{\text {neigh }}^{v c_{a c t}}$ to $v c_{a c t}$.

When the algorithm successfully allocates a VM node to $v c_{a c t}$, it stops the VCE step and starts a new iteration of 


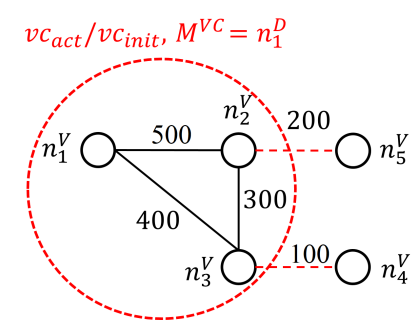

Fig. 9. After the $2^{\text {nd }}$ iteration of Phase II of the ViDCAlloc algorithm.

phase II while maintaining the current selection of $v c_{a c t}$. By following this procedure, ViDCAlloc essentially allocates as many VM nodes to a VC as possible in order to minimize the overall bandwidth requirements of the cross-VC VEs. If in some iteration none of the nodes in $N_{\text {neigh }}^{v c_{a c t}}$ can be allocated to $v c_{a c t}$, the algorithm starts the next step of phase II.

As an example, consider the $v c_{i n i t}$ shown in Fig. 6. In the first iteration of phase II, the ViDCAlloc algorithm starts the VCE step by selecting $v c_{i n i t}$ as $v c_{a c t}$. Then, the algorithm constructs $N_{n e i g h}^{v c_{a c t}}$ as $\left\{n_{2}^{V}, n_{3}^{V}\right\}$ and tries to allocate $n_{2}^{V}$ to $v c_{a c t}$ first. Because there is only one existing VC, $E_{\text {alloc }}$ is empty. Next, ViDCAlloc only performs the HC-Test and the OLC-Test for allocation $n_{2}^{V} \rightarrow v c_{a c t}$. Because this allocation passes both tests, the algorithm actually allocates $n_{2}^{V}$ to $v c_{a c t}$, i.e., allocates $n_{2}^{V}$ on server $n_{1}^{D}$. Fig. 8 shows the new $v c_{a c t}$, and $E_{\text {pend }}^{v c_{\text {act }}}$ (dashed red lines). In the second iteration, the ViDCAlloc algorithm selects $n_{3}^{V}$ with $d\left(n_{3}^{V}, v c_{a c t}\right)=700$ and allocates $n_{3}^{V}$ to $v c_{a c t}$. Fig. 9 shows the new $v c_{a c t}$ and $E_{\text {pend }}^{v c_{a c t}}$ (dashed red lines). In the third iteration, none of the nodes in $N_{n e i g h}^{v c_{a c t}}$ can be allocated to $v c_{a c t}$ due to lack of available CPU cores on the server $n_{1}^{D}$. As a result, the ViDCAlloc algorithm starts the next step in this iteration.

Step 2: New VC Establishment (NVCE) step. In this step, our goal is still to allocate one VM node and the related VEs. Since we have expanded each existing VC in previous iterations, we now consider allocating a VM node by establishing a new VC for it. The ViDCAlloc algorithm begins the NVCE step by identifying all pairs of an existing VC and its neighbor nodes. It then selects a pair $\left(n_{t}^{V}, v c_{p}\right)$ with the largest $d\left(n_{t}^{V}, v c_{p}\right)$. Since node $n_{t}^{V}$ cannot be added to $v c_{p}$ no matter which server we allocate $n_{t}^{V}$ to, we always need to allocate all VEs that connect $n_{t}^{V}$ and $v c_{p}$. We then try to allocate $n_{t}^{V}$ on a server that is close to $M^{V C}\left(v c_{p}\right)$ in order to minimize the length of the paths allocated for the VEs that connect $n_{t}^{V}$ and $v c_{p}$. To achieve this goal, the ViDCAlloc algorithm sorts each $n_{i}^{D}$ in $N^{D}$ (except $M^{V C}\left(v c_{p}\right)$ ) in increasing order of the distance (number of hops) between $n_{i}^{D}$ and $M^{V C}\left(v c_{p}\right)$. If some nodes have the same distance to $M^{V C}\left(v c_{p}\right)$, the algorithm sorts them in decreasing order of their resource availability. Then, for each node $n_{i}^{D}$, the ViDCAlloc algorithm examines the feasibility of allocating $n_{t}^{V}$ on that node.

For an allocation $n_{t}^{V} \rightarrow n_{i}^{D}$, there are two cases. The first case is that some existing $\mathrm{VC} v c_{e}$ is already allocated on server $n_{i}^{D}$. In such a case, ViDCAlloc tries to allocate $n_{t}^{V}$ to $v c_{e}$. To do so, it repeats the procedure introduced in the VCE step for the allocation $n_{t}^{V} \rightarrow v c_{e}$. The second case is that there is no existing VC allocated on $n_{i}^{D}$. In such a case, ViDCAlloc creates a new VC $v c_{n e w}$ which only contains $n_{t}^{V}$ and tries to allocate $v c_{n e w}$ on $n_{i}^{D}$. The algorithm constructs $E_{\text {alloc }}$

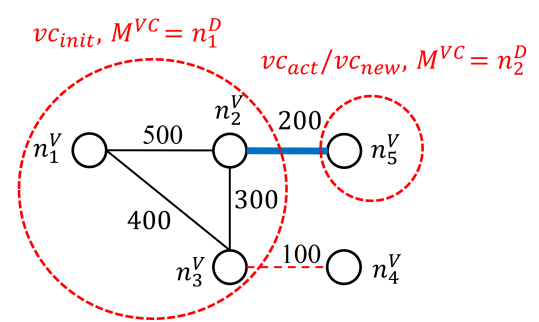

Fig. 10. After the $3^{\text {rd }}$ iteration of Phase II of the ViDCAlloc algorithm.

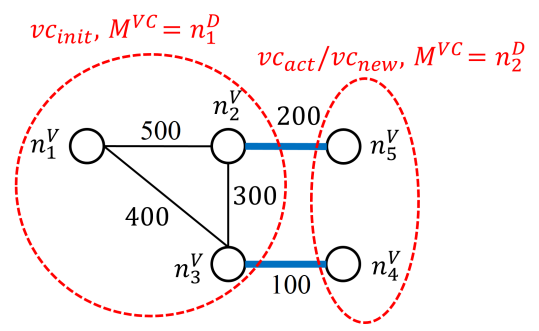

Fig. 11. After the $4^{\text {th }}$ iteration of Phase II of the ViDCAlloc algorithm.

and $E_{\text {pend }}^{v c_{\text {new }}}$. Then, it performs the tests VEA-Test, HC-Test, and OLC-Test for allocation $v c_{n e w} \rightarrow n_{i}^{D}$. If the allocation passes all tests, ViDCAlloc actually allocates $v c_{\text {new }}$ on server $n_{i}^{D}$ and reserves bandwidth for each VE in $E_{\text {alloc }}$ along the corresponding physical path found in the VEA-Test.

If the ViDCAlloc algorithm successfully allocates the selected node $n_{t}^{V}$, it then stops the NVCE step and starts a new iteration by selecting $v c_{e}$ or $v c_{n e w}$ as $v c_{a c t}$. Furthermore, ViDCAlloc has an early termination criterion: If the algorithm tries to allocate $n_{t}^{V}$ on an empty server, i.e., a server that is not hosting any VMs of any user, it stops the NVCE step. The intuition here is that if an empty server cannot accommodate $n_{t}^{V}$, it is highly likely that none of the servers can accommodate $n_{t}^{V}$. This criterion effectively reduces the runtime of the ViDCAlloc algorithm, which we demonstrate through simulations in section V. If ViDCAlloc cannot allocate $n_{t}^{V}$ on any server at the end of the NVCE step, it stops and rejects the input request. However, if ViDCAlloc has successfully allocated all VM nodes at the end of an iteration, it then accepts the user request and returns $M^{N}$ and $M^{E}$.

For the previous example, in the third iteration, the ViDCAlloc algorithm starts the NVCE step with selecting the pair $\left(n_{5}^{V}, v c_{a c t}\right)$. Next, it tries to allocate $n_{5}^{V}$ on each node $n_{i}^{D}$ following sequence $\left(n_{2}^{D}, n_{4}^{D}, n_{3}^{D}\right)$. For the first allocation $n_{5}^{V} \rightarrow n_{2}^{D}$, because there is no existing $\mathrm{VC}$ on $n_{2}^{D}$, the algorithm creates a new VC $v c_{\text {new }}$ and examines the feasibility of allocating $v c_{\text {new }}$ on $n_{2}^{D}$. Fig. 10 shows the new VC $v c_{\text {new }}$. After constructing $E_{\text {alloc }}$ as $\left\{e_{25}^{V}\right\}$ (bold blue line in Fig. 10), the algorithm performs the tests VEA-Test, HC-Test, and OLCTest. In the VEA-Test, the algorithm finds the path $\left(e_{15}^{D}, e_{25}^{D}\right)$ for $\operatorname{VE} e_{25}^{V}$. Because $v c_{\text {new }}$ passes all tests, the algorithm actually allocates $v c_{\text {new }}$ and reserves bandwidth for $e_{25}^{V}$ along the path $\left(e_{15}^{D}, e_{25}^{D}\right)$. Then, ViDCAlloc selects $v c_{n e w}$ as $v c_{a c t}$ and starts a new iteration. In the fourth iteration, since $N_{\text {neigh }}^{v c_{a c t}}$ is empty, the algorithm skips the VCE step and starts the NVCE step by selecting pair $\left(n_{4}^{V}, v c_{1}\right)$. Then, it first tries to allocate $n_{4}^{V}$ on server $n_{2}^{D}$. Because $v c_{a c t}$ is on $n_{2}^{D}$, the algorithm allocates $n_{5}^{V}$ to $v c_{a c t}$ and path $\left(e_{15}^{D}, e_{25}^{D}\right)$ for $e_{34}^{V}$. 


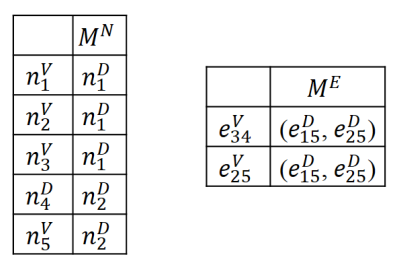

Fig. 12. The output of ViDCAlloc for the input shown in Figs. 2(a) and 3(a).

Fig. 11 shows the new $v c_{a c t}$. After the fourth iteration, all VMs have been allocated. As a result, ViDCAlloc finishes with an objective value of $600 \mathrm{Mbps}$. The returned mapping $M^{N}$ and $M^{E}$ is shown in Fig. 12.

\section{Runtime Complexity}

Assume that the number of VM nodes in $G^{V}$ is $\left|N^{V}\right|$; the number of VEs is $\left|E^{V}\right|$; the number of nodes in $G^{D}$ is $\left|N^{D}\right|$; and the number of physical links in $G^{D}$ is $\left|E^{D}\right|$. The worst case runtime complexity of the ViDCAlloc algorithm can be written as:

$$
O\left(\left|N^{V}\right| \cdot\left|E^{V}\right| \cdot\left|N^{D}\right|^{2} \cdot \log \left(\left|N^{D}\right|\right)+\left|N^{V}\right|^{3}\right)
$$

We omit the procedure of arriving at Eq. 7 due to space limitations. Note that, in practice, the value of $\left|N^{D}\right|$ and $\left|E^{D}\right|$ is usually much larger than $\left|N^{V}\right|$ and $\left|E^{V}\right|$. For example, traces of workloads running on a Google compute cell [13] have shown that while the Google computing cell consists of 12000 servers, most of user jobs request less than 50 machines. As a result, $\left|N^{D}\right|^{2} \cdot \log \left(\left|N^{D}\right|\right)$ usually dominates the complexity of the ViDCAlloc algorithm.

\section{Performance Evaluation}

\section{A. Simulation Setup}

We evaluate the ViDCAlloc algorithm through a set of online simulations. In each simulation, we generate a set of VDC requests, denoted by $\mathcal{R}$. Each request $r_{i} \in \mathcal{R}$ has its own start time $s t_{i}$ and lifetime $l t_{i}$. At the start time of each request, we run the ViDCAlloc algorithm to service the request. Whenever a request is serviced/finished, we reduce/restore the reserved resources from/to the data center.

We generate the VDC requests based on statistics of traces of workloads running on an 12000-server Google compute cell over a period approximately a month long, in May 2011 [13]. We exclude jobs that require only one server, since servicing them is trivial. Fig. 13 shows the histogram of job start times in a 24-hour scale. In our simulations, we use this statistical data as the probability distribution to generate the start time of each request. We also observe that while $90 \%$ of jobs require less than $50 \mathrm{VMs}$, a few jobs require as many as $2000 \mathrm{VMs}$. Based on this observation, we chose to use in our simulations the Weibull distribution with shape parameter $k=0.8$ and scale parameter $\lambda=0.2$ to approximate the real distribution of the number of VMs requested by each user. Fig. 14 shows the probability density function (PDF) of the used Weibull distribution. The lifetime of each request is determined by an exponential distribution with mean $\mu=90$ minutes, following similar setups found in existing works [8], [12]. The number of CPU cores required by each VM is randomly selected between 1 and 3. Furthermore, we use the random graph model

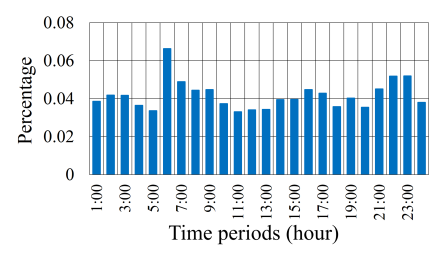

Fig. 13. Start time of jobs submitted to the Google computing cell in May'11.

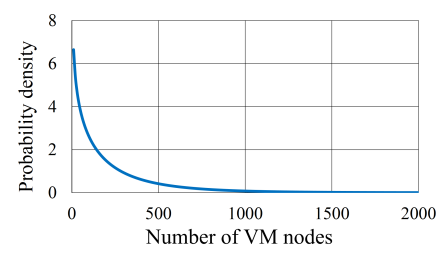

Fig. 14. PDF of the Weibull distribution used to generate the input.

proposed by Waxman [20] to generate the virtual topology. In this model, $N$ nodes are distributed in the $\mathrm{x}-\mathrm{y}$ coordinate plane, and the probability of an edge existing between node $u$ and $v$ is given by:

$$
P(u, v)=\beta \exp \left(\frac{-d(u, v)}{L \alpha}\right)
$$

where $d(u, v)$ is the distance between $u$ and $v, L$ is the maximum distance between two nodes, and $\alpha$ and $\beta$ are parameters in the range $(0,1]$. A small value of $\alpha$ results in higher density of short edges relative to longer ones, while a large $\beta$ value increases edge density. In our simulations, we set $\alpha=0.1$ and $\beta=0.05$. Furthermore, the bandwidth requirement of each VE is randomly selected between 0 and 100 Mbps.

For the physical data center, we use a FatTree [21] architecture. A $k$-array FatTree network has $k$ pods, where each pod has $k / 2$ Top-of-Rack (ToR) switches and $k / 2$ aggregation switches. While in each pod the ToR and aggregation switches are connected to each other as a complete bipartite graph, each ToR switch also connects a rack of $k / 2$ hosts. In addition, there are $(k / 2)^{2}$ core switches that connect the aggregation switches of all pods. In general, a $k$-array fat-tree network is able to support $k^{3} / 4$ hosts. In our simulations, the number of available CPU cores of each host is 10 , and the bandwidth capacity of intra-rack links and inter-rack links is $1 \mathrm{Gbps}$ and $10 \mathrm{Gbps}$ respectively.

We compare the ViDCAlloc algorithm with LoCo [12] and SecondNet [3]. The LoCo algorithm [12] collocates VMs on the same host and runs in iterations. In each iteration, LoCo identifies the VE that connects an unmapped node $u$ to a mapped node $v$ with the largest bandwidth requirement. Subsequently, it tries to map $u$ on the host of $v$. If the mapping succeeds, LoCo maps all VEs that connects $u$ to any mapped node. Note that the pre-clustering approach proposed in [12] solves a MIP problem, which has a high complexity. For example, to address a VDC request with just $100 \mathrm{VM}$ nodes, the pre-clustering approach takes approximately 700 seconds to cluster the VDC request, while the LoCo algorithm only takes approximately 10 seconds to address the clustered VDC request. For 200 VM nodes the pre-clustering approach takes roughly 4.5 hours while LoCo only takes approximately 
50 seconds. $^{3}$ Due to the complexity of such a solution, we consider it impractical for large scale problems and we chose not to use it in our simulations. The SecondNet algorithm [3] allocates VMs on physical servers in a one-on-one mapping manner. It groups servers into multiple clusters and tries to allocate resources for the requested VDC in each such cluster in increasing order of cluster size. SecondNet formulates and solves the node allocation problem as a max-min cost flow problem. After allocating the VM nodes, it finds a physical path for each VE and reserves bandwidth along the corresponding path. In case of allocation failure, SecondNet tries to allocate the VDC on the next cluster.

\section{B. Evaluation Metrics}

We use four metrics to evaluate the performance of the ViDCAlloc algorithm:

(a) The number of accepted requests. The number of requests accepted by an algorithm is an important metric to evaluate the performance of that algorithm. However, this number cannot completely represent the performance of an algorithm when the ultimate goal of a cloud is to generate more revenue. An algorithm can increase the number of accepted requests by accepting many smaller requests, which are usually less profitable.

(b) Total generated revenue. We also measure the total revenue generated by an algorithm. We adopt the typical "payas-you-go" revenue model used in clouds to define the revenue of a request. We can express it as:

$$
\left(\alpha \cdot \sum_{n_{i}^{V} \in r_{k}} r\left(n_{i}^{V}\right)+\beta \cdot \sum_{e_{i j}^{V} \in r_{k}} r\left(e_{i j}^{V}\right)\right) \cdot l t_{r}
$$

where $l t_{r}$ is lifetime of the request, and $\alpha$ and $\beta$ are the unit price charged for computing and bandwidth resources. The total revenue is then defined as the sum of the revenue of each accepted request. In our simulations, we set $\alpha=\beta=1$.

(c) Total generated cost. We measure the total cost generated by an algorithm to service the submitted requests. First, we define the cost of an accepted request as:

$$
\left(\sum_{n_{i}^{V} \in r_{k}} r\left(n_{i}^{V}\right)+\sum_{e_{i j}^{V} \in E_{C}^{V}} \sum_{e_{p q}^{D} \in M^{E}\left(e_{i j}^{V}\right)} r\left(e_{i j}^{V}\right)\right) \cdot l t_{r}
$$

Then, the total cost is the sum of cost of each accepted request. Given the objective function of the ViDCAlloc algorithm, as shown in Eq. 5, the total generated cost also gives an idea of how well the ViDCAlloc algorithm performs in achieving its objective.

(d) Average running time. Finally, we measure the average running time of an algorithm to address a request in the set $\mathcal{R}$. As mentioned previously, a cloud may comprise a large number of servers and the SP of this cloud may need to service a vast amount of requests in a short period. As a result, the running time of the scheduling algorithm is a critical factor that affects the number of users serviced by a cloud.

\footnotetext{
${ }^{3}$ The result is an average of ten simulation runs performed on an Intel Xeon E5-2650 2.00 GHz processor.
}

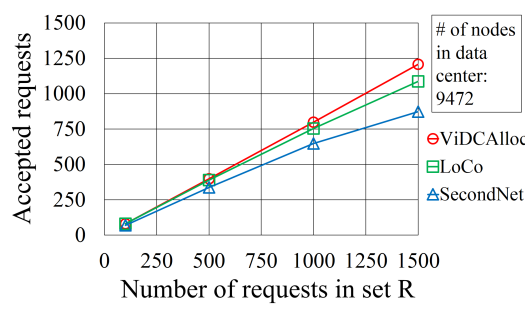

Fig. 15. Results of the first simulation: Accepted requests.

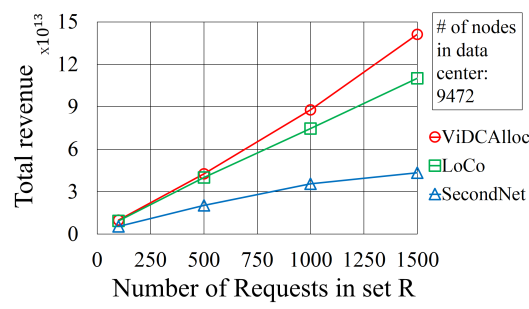

Fig. 16. Results of the first simulation: Total revenue.

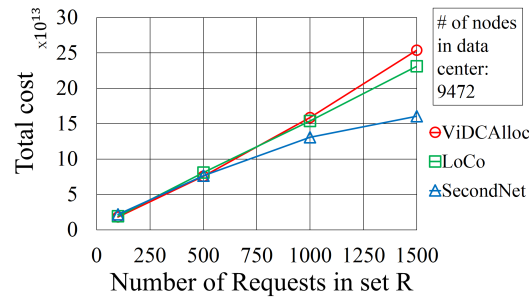

Fig. 17. Results of the first simulation: Total cost.

\section{Performance with Increasing Number of Requests}

In this simulation, we study how the ViDCAlloc algorithm performs as the number of requests in set $\mathcal{R}$ increases from 100 to 1500 . Each request is generated by using the way introduced in Section V-A. For the physical data center, we use a 32-array FatTree architecture that has 9472 nodes composed by 8192 servers and 1280 switches. Each data point in our simulation results is an average of ten simulations performed on an Intel Xeon E5-2650 2.00 GHz processor.

Fig. 15 shows the number of accepted requests. While all three algorithms accept similar number of requests when the number of input requests is small, the ViDCAlloc algorithm accepts more requests than other algorithms as the number of requests increases. When the number of input requests is 1500, ViDCAlloc accepts $12 \%$ more requests than LoCo and nearly $40 \%$ more than SecondNet. Fig. 16 shows the total revenue generated by each algorithm. We can see that the ViDCAlloc algorithm generates the most revenue, followed by LoCo and SecondNet. When the number of input requests is 1500 , ViDCAlloc generates nearly $30 \%$ more revenue than LoCo and nearly $220 \%$ more revenue than SecondNet. This is because ViDCAlloc accepts a larger number of large requests, which generate more revenue. As an example, Fig. 19 shows the acceptance rate of requests with different sizes, when the set $\mathcal{R}$ contains 1000 requests and the data center has 9472 nodes. We observe that when the number of VM nodes in the requests is larger than 1000 , the acceptance rate of ViDCAlloc is $15 \%-135 \%$ higher than that of LoCo and 15-30 times higher 


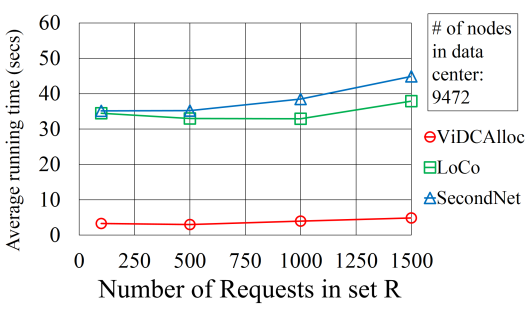

Fig. 18. Results of the first simulation: Average running time.

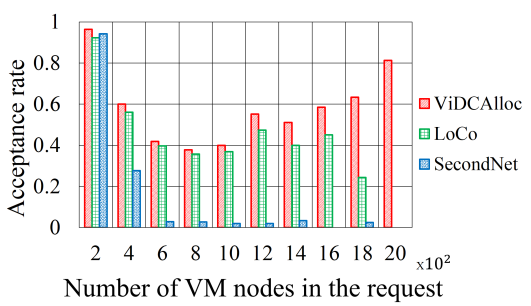

Fig. 19. Acceptance rate of requests with different sizes in the simulations in which $\mathcal{R}$ contains 1000 requests and the data center comprises 9472 nodes.

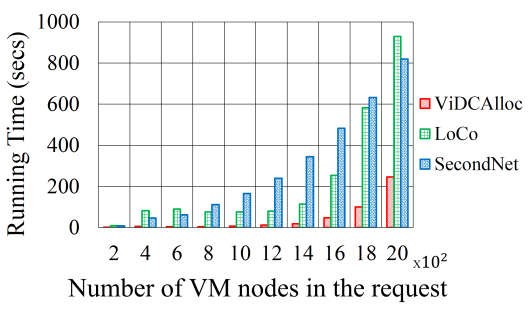

Fig. 20. Average running time to address requests with different sizes in the simulations in which $\mathcal{R}$ contains 1000 requests and the data center comprises 9472 nodes.

than that of SecondNet. When the number of VM nodes in the requests is in the range 1800-2000, the acceptance rate of ViDCAlloc is $80 \%$, while that of LoCo and SecondNet is 0 . Furthermore, Fig. 17 shows the total cost generated by each algorithm. When the number of requests is smaller than 1000 , the three algorithms generate similar total cost; when the number of requests is 1500 , ViDCAlloc generates $10 \%$ more cost than LoCo and 55\% more cost than SecondNet, because the ViDCAlloc algorithm accepts more requests than LoCo and SecondNet. From Fig. 15, 16, 17 and 19, we can observe that ViDCAlloc achieves higher acceptance rate and larger revenue by accepting more large size requests. We attribute this to ViDCAlloc having better strategies on collocating VMs and allocating $\mathrm{VCs}$, which reduce the bandwidth requirements to be reserved and shorten the length of allocated paths. Such strategies reduce the cost of allocating an individual request, and thereby retain more available resources to accommodate more of the requests that follow.

Finally, Fig. 18 shows the average running time each algorithm takes to address a request. We can see that the ViDCAlloc algorithm is generally 10 times faster than LoCo and SecondNet, i.e., ViDCAlloc takes approximately 4 seconds vs. 35-40 seconds taken by LoCo and SecondNet. This is because ViDCAlloc is faster in addressing large size requests. As an example, Fig. 20 shows the average running time of each algorithm in addressing requests of different size, when the set $\mathcal{R}$ has 1000 requests and the data center comprises 8192 servers. While ViDCAlloc takes 100-200 seconds to

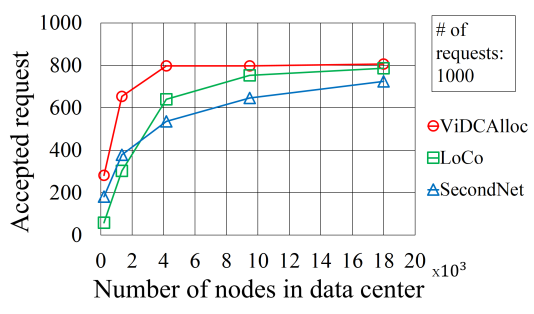

Fig. 21. Results of the second simulation: Accepted requests.

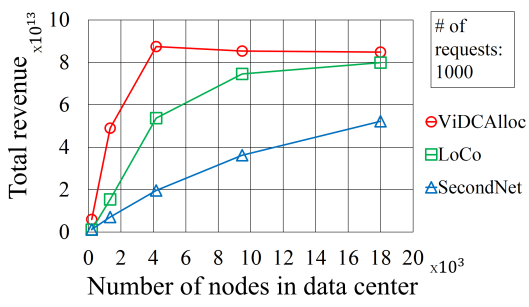

Fig. 22. Results of the second simulation: Total revenue.

address requests that have more than $1000 \mathrm{VM}$ nodes, LoCo and SecondNet take 300-900 seconds to address such requests. We attribute this to ViDCAlloc having a better strategy for estimating the feasibility of servicing a request, and thereby rejecting requests with low possibility of accommodation earlier (line 29 in Fig. 7). On the other hand, the better performance of ViDCAlloc proves that the technique of early termination does not have an adverse impact on the efficiency of the algorithm.

\section{Performance with Increasing Size of Data Center}

We now demonstrate the performance of the ViDCAlloc algorithm as the size of the data center increases. We increase the number of pods in the FatTree architecture from 8 to 40 with an increment value of 8 pods. As a result, the number of nodes in the data center increases from 208 to 18000 . The number of requests in set $\mathcal{R}$ is 1000 . Each data point in our simulation results is an average of ten simulations performed on an Intel Xeon E5-2650 2.00 GHz processor.

Fig. 21 shows the number of accepted requests. We can see that the ViDCAlloc algorithm accepts approximately 5\%$115 \%$ more requests than LoCo and $10 \%-75 \%$ more requests than SecondNet. We also observe that the number of requests accepted by ViDCAlloc almost remains the same when the size of data center exceeds 4000 nodes. A possible reason is that during certain time periods the total resource requirements of all submitted requests may be much larger than the total available resources in the data center; therefore, although the size of data center increases, we still cannot accept more requests during those time periods. In other words, ViDCAlloc has accepted almost all requests that can be possibly accepted. Fig. 22 shows the total revenue generated by each algorithm. ViDCAlloc generates 10\%-200\% more revenue than LoCo and 65\%-350\% more than SecondNet. Fig. 23 shows the total cost generated by each algorithm. ViDCAlloc generates more cost in the beginning, due to its much higher acceptance rate; as the size of data center increases, the cost generated by ViDCAlloc is actually decreasing. When data center has 18000 nodes, the total cost generated by ViDCAlloc is around $10 \%$ less than that of LoCo and around 25\% less than that of SecondNet. We attribute this to ViDCAlloc having better strategies for allocating paths for VEs. As the size of data center increases, 


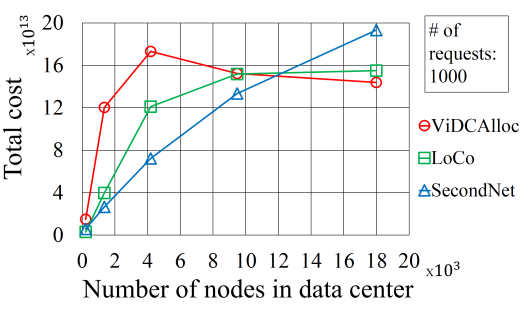

Fig. 23. Results of the second simulation: Total cost.

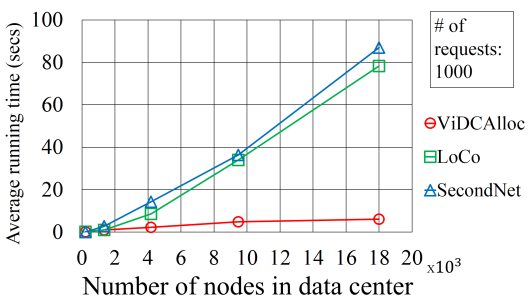

Fig. 24. Results of the second simulation: Average running time.

the number of servers in each rack and pod also increases. The ViDCAlloc algorithm then utilizes more intra-rack and intra-pod links to allocate VEs. Consequently, such strategies reduce the length of allocated paths and decrease the total cost. Finally, Fig. 24 shows the average running time each algorithm takes to address a single request. Again, we observe that the ViDCAlloc algorithm is up to 12 times faster than LoCo and SecondNet. As discussed previously, the reason is that the ViDCAlloc algorithm is much faster when addressing largesize requests. An example has already been given in Fig. 20. Note that when the data center comprises 18000 nodes, given the average running time shown in Fig. 24, the SecondNet algorithm takes more than 24 hours to address 1000 requests submitted in a 24 -hour period.

\section{CONCLUSion}

In this paper, we studied the problem of allocating virtual data centers in the physical data centers of clouds called the VDCA problem. We captured the important differences between the VDCA and the VNE problem, and decomposed VDCA into three sub-problems: VDC clustering, VM allocation, and virtual link allocation. We proved the NP-hardness of VDCA and proposed an algorithm called ViDCAlloc to solve it. The ViDCAlloc algorithm jointly solves the three sub-problems and minimizes the total amount of bandwidth reserved for the virtual data center.

We studied the performance and scalability of the ViDCAlloc algorithm through a set of online simulations. In the simulations, we generated the input based on the traces of workload running on a Google compute cell and compare ViDCAlloc with two existing algorithm, called LoCo and SecondNet. Simulation results show that the ViDCAlloc algorithm accepts 5\%-115\% more requests than LoCo and $10 \%-75 \%$ more requests than SecondNet. The ViDCAlloc algorithm also generates 30\%-200\% more revenue than LoCo and $55 \%-300 \%$ more revenue than SecondNet. Simulation results also show excellent scalability of the ViDCAlloc algorithm. Generally, the ViDCAlloc algorithm can be up to 10-12 times faster than LoCo and SecondNet. In a data center having 18000 machines, while the average running time of LoCo and SecondNet to address a request is approximately 80 seconds, the average running time of the ViDCAlloc algorithm is only 6 seconds. In addition to that, for large-size requests with more than $1000 \mathrm{VMs}$, the ViDCAlloc algorithm takes an average of $100-200$ seconds vs. an average of 300-900 seconds taken by LoCo and/or SecondNet.

\section{REFERENCES}

[1] “Amazon EC2," http://aws.amazon.com/ec2

[2] "Microsoft Azure," https://azure.microsoft.com

[3] C. Guo, G. Lu, H. Wang, S. Yang, C. Kong, P. Sun, W. Wu, and Y. Zhang, "Secondnet: a data center network virtualization architecture with bandwidth guarantees," in Proc. of ACM CoNEXT 2010, Philadelphia, PA, November 30-December 3, 2010.

[4] H. Ballani, P. Costa, T. Karagiannis, and A. Rowstron, "Towards predictable datacenter networks," ACM SIGCOMM Computer Communication Review, vol. 41, no. 4, pp. 242-253, 2011.

[5] S. Radhakrishnan, R. Pan, A. Vahdat, and G. Varghese, "Netshare and stochastic netshare: predictable bandwidth allocation for data centers," ACM SIGCOMM Computer Communication Review, vol. 42, no. 3, pp. 5$11,2012$.

[6] L. Popa, P. Yalagandula, S. Banerjee, J. C. Mogul, Y. Turner, and J. R. Santos, "Elasticswitch: practical work-conserving bandwidth guarantees for cloud computing," in Proc. of the ACM SIGCOMM 2013, Hong Kong, China, August 12-16, 2013.

[7] A. Fischer, J. Botero, M. Beck, H. De Meer, and X. Hesselbach, "Virtual network embedding: A survey," Communications Surveys \& Tutorials, no. 4, pp. 1888-1906, 2013.

[8] M. Chowdhury, M. R. Rahman, and R. Boutaba, "Vineyard: Virtual network embedding algorithms with coordinated node and link mapping," IEEE/ACM Transactions on Networking (TON), vol. 20, no. 1, pp. 206-219, 2012.

[9] M. Yu, Y. Yi, J. Rexford, and M. Chiang, "Rethinking virtual network embedding: substrate support for path splitting and migration," $A C M$ SIGCOMM Computer Communication Review, vol. 38, no. 2, pp. 17-29, 2008.

[10] J. Lischka and H. Karl, "A virtual network mapping algorithm based on subgraph isomorphism detection," in Proc. of the 1st ACM workshop on Virtualized infrastructure systems and architectures, Barcelona, Spain, August 17, 2009.

[11] L. Gong, Y. Wen, Z. Zhu, and T. Lee, "Toward profit-seeking virtual network embedding algorithm via global resource capacity," in Proc. of IEEE INFOCOM 2014, Toronto, Canada, April 27-May 2, 2014.

[12] C. Fuerst, S. Schmid, and A. Feldmann, "Virtual network embedding with collocation: Benefits and limitations of pre-clustering," in Proc. of IEEE Cloud Networking (CloudNet) 2013, San Francisco, CA, November 11-13, 2013.

[13] J. L. Hellerstein, W. Cirne, and J. Wilkes, "Google cluster data," Google research blog, Jan, 2010.

[14] C. Papagianni, A. Leivadeas, S. Papavassiliou, V. Maglaris, C. CervelloPastor, and A. Monje, "On the optimal allocation of virtual resources in cloud computing networks," IEEE Transactions on Computers, vol. 62, no. 6, pp. 1060-1071, 2013.

[15] "The Xen project," http://www.xenproject.org/

[16] “The KVM project," http://www.linux-kvm.org/

[17] E. G. Coffman Jr, M. R. Garey, and D. S. Johnson, "Approximation algorithms for bin packing: A survey," in Approximation algorithms for NP-hard problems, pp. 46-93, 1996.

[18] D. Johnson, A. Demers, J. Ullman, M. Garey, and R. Graham, "Worstcase performance bounds for simple one-dimensional packing algorithms," SIAM Journal on Computing 3, no. 4, pp. 299-325, 1974.

[19] E. Dijkstra, "A note on two problems in connexion with graphs," Numerische mathematik 1, no. 1, pp. 269-271, 1959.

[20] B. M. Waxman, "Routing of multipoint connections," Selected Areas in Communications, IEEE Journal on, vol. 6, no. 9, pp. 1617-1622, 1988.

[21] M. Al-Fares, A. Loukissas, and A. Vahdat, "A scalable, commodity data center network architecture," ACM SIGCOMM Computer Communication Review, vol. 38, no. 4, pp. 63-74, 2008. 